\section{Wahrnehmung, Bewertung und die Akzeptabilität von Technik in Deutschland}

\author{
Die Problematik der Erfassung von \\ Technikeinstellungen am Beispiel von \\ externer Technik und Gentechnik
}

von Jürgen Hampel und Michael M. Zwick, Institut für Sozialwissenschaften, Universität Stuttgart

Sind die Deutschen technikfeindlich? Diese Frage erscheint in dieser Form nicht mehr zeitgemäß. Die empirische sozialwissenschaftliche Forschung hat ein bemerkenswert differenziertes Bild der Beurteilung von Technik in der Öffentlichkeit ermittelt. Zustimmung oder Ablehnung treffen nicht Technik als solche, sondern ganz konkrete Technologien und deren Anwendung. Ähnlich vielgestaltig wie die Bewertung unterschiedlicher Technologien sind die Gründe für Zustimmung oder Ablehnung. Maßgeblich sind Kriterien wie die wahrgenommenen Nutzen- und Schadenspotenziale und ihre Verteilung, ethische Unbedenklichkeit, die Einschätzung einer angemessenen Regulierung sowie das Vertrauen in Entwickler, Betreiber und Regulatoren. Daneben verlangt die Bevölkerung in wachsendem Maße nach direkter Mitwirkung bei technikbezogenen Entscheidungen.

Do Germans have a hostile attitude towards technology? This question does not seem current anymore. Empirical social-scientific research has identified a remarkably distinguished public image of technology. Approval or disapproval does not refer to technology as such, but to specific technologies and their application. And the reasons for approval or disapproval are as manifold as the assessment of different technologies. Relevant criteria are the perceived benefits and risks, ethical acceptability, the estimation of a reasonable regulation as well as trust in developers, operators and regulators. In addition, the public is increasingly asking for direct involvement in decisions on technologies.

\section{Einleitung}

Warum interessiert Forschung zur Wahrnehmung, Bewertung und Akzeptanz von Technik? Das Thema „Technikkommunikation“ hat in den vergangenen Jahren erheblich an Bedeutung gewonnen. Dass über Technik mit der Öffentlichkeit kommuniziert werden muss, ist eine weitgehend unbestrittene Selbstverständlichkeit (Weitze/Heckl 2016). Kommunikation ist aber nicht voraussetzungslos. Voraussetzung jeder Kommunikation ist die Kenntnis des Gegenübers. Während in der direkten Kommunikation zwischen Anwesenden Missverständnisse in einem iterativen Prozess ausgeräumt werden können, beruhen nichtinteraktive Formen der Kommunikation entweder auf impliziten Annahmen über die Kommunikationspartner oder auf empirisch fundiertem Wissen über die Gründe und Begründungen, die die Haltung eines Gegenübers zu einem Gegenstand bestimmen. Diese Annahmen, die richtig, aber auch falsch sein können, bestimmen, worüber kommuniziert wird. Wenn diese Annahmen falsch sind, produzieren Kommunikationsanstrengungen nichts als Rauschen.

Mit unserem Beitrag wollen wir daher die empirische Forschung zum Thema Technikeinstellungen skizzieren und sie zugleich mit ausgewähltem empirischem Material untermauern. Damit soll auch herausgearbeitet werden, was die empirische Forschung zum Thema Technikeinstellungen empirisch an Gründen für die Unterstützung oder Ablehnung von Technik ermittelt hat. Dieses Unternehmen bleibt zwangsläufig selektiv: $\mathrm{Zu}$ vielgestaltig ist, was wir als Technik erleben.

Die Verschiedenartigkeit von Technik und ihren gesellschaftlichen Auswirkungen hat zugleich einen Gestaltwandel der Fragen nach Technikakzeptanz bewirkt. An die Stelle von Globalindikatoren tritt eine zunehmend differenzierte Erfassung von Technikeinstellungen. Mit immer feineren Optiken gilt es, nicht nur einzelne Technologien, sondern, wie etwa bei Gen- oder Nanotechnik, verschiedene Anwendungsfelder unter die Lupe zu nehmen, die in der Öffentlichkeit unterschiedlich wahrgenommen und beurteilt werden, je nachdem, welche Risiken und Nutzenpotenziale, welche Folgen für Gesellschaft, Wirtschaft, Um- 
welt und Gesundheit vermutet und wie diese bewertet werden. Aber auch ob und wenn ja, welche ethischen Sensibilitäten berührt werden, wie es gegebenenfalls um die institutionelle Regulierung und Kontrolle von Technik bestellt ist, und ob die Mitwirkungsmöglichkeiten der Öffentlichkeit bei der Implementation von Groß- und infrastrukturellen Projekten wahrgenommen und die erzielten Arrangements als transparent und fair empfunden werden, kann für die Akzeptabilität einer Technik ausschlaggebend sein.

Ausgehend von den Studentenunruhen der späten 1960er und der sozialen Bewegungsepoche der 1970er Jahre hat sich in den letzten Dekaden das politische Selbstbewusstsein der Bürger verstärkt und u. a. zu einer Neubestimmung dessen geführt, was wir unkritisch als „Technikakzeptanz“ eingeführt haben. Nicht nur Groß- und infrastrukturtechnische Projekte werden kritischer gesehen - an neuen Technologien entzünden sich auch Wertkonflikte. Legitimation durch formale Entscheidungsprozesse hat sich als nicht mehr ausreichend erwiesen, daher bedürfen Technisierungsprozesse heute einer breiten gesellschaftlichen Legitimation. Bleibt diese aus, drohen effektive Politisierungs- und Mobilisierungsprozesse, Bürgerinitiativen, Prozesslawinen und nicht selten das Aus für geplante Projekte. Nicht länger geht es um Technikakzeptanz, verstanden als Imperativ, missliebige Technik- und Infrastrukturprojekte zähneknirschend hinzunehmen, sondern darum, dialogisch Bedingungen herzustellen, unter denen solche Projekte für die Betroffenen akzeptabel sind (vgl. Grunwald 2008; acatech 2011, S. 7).

\section{Zur Geschichte der Erforschung von Technikeinstellungen}

Joseph Huber hat in seinem Buch „Technikbilder“ (1989) gezeigt, dass kontroverse Bewertungen von Technik nicht erst ein Phänomen der Gegenwart sind, sondern bis in die Zeiten der Aufklärung zurückreichen, die auch die Zeit der beginnenden industriellen Revolution war. Kritische Traditionen lassen sich über die Jugendbewegung der Wende vom 19. zum 20. Jahrhundert, den deutschen Idealismus und die Romantik bis zurück zu Jean Jacques Rousseau verfolgen.
Die Problematisierung von Technikeinstellungen und deren empirische Messung sind ein Produkt des Kalten Kriegs. 1957 war das Jahr des Sputnik-Schocks, bei dem die bis dahin als fraglos angenommene technische Überlegenheit der USA grundlegend erschüttert wurde. Im gleichen Jahr wurde die erste empirische Studie zum Thema „Einstellungen zu Wissenschaft und Technik“ von der US-amerikanischen National Association of Science-Writers, der Vereinigung der amerikanischen Wissenschaftsjournalisten durchgeführt. Das amerikanische „Public Understanding of Science“-Programm mit dem Ziel, Wissenschaft zu popularisieren, nahm seine Arbeit auf (Miller 2004). Diese frühe Studie blieb allerdings noch ohne direkte Nachfolger. Es dauerte noch einmal bis in die späten 1970er Jahre, bis in den USA Technikeinstellungen systematisch erforscht wurden. Seither sind Indikatoren zu Technikeinstellungen integraler Bestandteil des Sachstandsberichts der National Science Foundation zur technischen und wissenschaftlichen Entwicklung der USA, die Science and Engineering Indicators (National Science Board 2014).

Diesseits des Atlantiks hat die Europäische Union europaweite Eurobarometerbefragungen zur Erfassung von Einstellungen zu Technik und Wissenschaft durchgeführt, zum ersten Mal 1989, zuletzt 2015. Ferner liegen einige Spezialeurobarometer-Erhebungen vor, etwa zu dem Thema Biotechnology (in den Jahren 1991, 1993, 1996, 1999, 2002, 2005 und 2010).

\section{Messung von Technikeinstellungen und Differenzierung von Technikbereichen}

\subsection{Fluch oder Segen der modernen Technik? Technikeinstellungen im Spiegel eines Globalindikators}

Technikeinstellungen zählen zu jenen Phänomenen, die sich einer direkten, ,geeichten“ Messung entziehen (Hennen 1994, S. 5). Es bedarf geeigneter Indikatoren, um die Einstellungen der Menschen gegenüber „Technik“ messen zu können. Dabei müssen zwei Probleme unterschieden werden: Was messen wir? Wie messen wir?

Es versteht sich von selbst, dass das Antwortverhalten auf Fragen nicht nur vom Zielob- 
jekt abhängt, welches erfragt wird, sondern auch von der jeweiligen Formulierung der Frage. Mit anderen Worten: Die quantitative Verteilung des Antwortverhaltens ist bei einmaliger Befragung mitunter nur schwer interpretierbar, kann aber bei identischer Frageformulierung im Zeitverlauf besonders interessante Einblicke versprechen und Trends erkennen lassen.

Die Frage nach „Technikeinstellungen“ wurde in Deutschland erstmals in den 1960er Jahren virulent, nachdem Technik nach dem Zweiten Weltkrieg fraglos bejaht wurde, führte sie doch nicht nur zum wirtschaftlichen Wiedererstarken Deutschlands. Technik trug mit dazu bei, Wohlstand für viele zu schaffen - Stichwort „Wirtschaftswunder“ - und nebenbei die internationale Reputation Deutschlands zu verbessern. „Made in Germany" stand für attraktive Produkte und versprach solide Qualität.

In den 1960er Jahren, verstärkt aber seit den 1970er Jahren, mischten sich allerdings kritische Töne in die übliche Technikeuphorie, die den Ruf nach der empirischen Erforschung der Technikeinstellungen in Deutschland auslösten. In der Folge wurde eine Reihe von globalen Indikatoren entwickelt, um Zeitreihen von Bilanzurteilen über „Technik“ zu erhalten (Renn/Zwick 1997, S. 18).

Die längste Zeitreihe lässt sich für den renommierten „Fluch-Segen“-Indikator des Instituts für Demoskopie in Allensbach darstellen, die nach Auskunft des IfD erst $2011 \mathrm{ihr}$ (vorläufiges) Ende fand. In Abbildung 1 sind die Anteile der Antworten (ohne fehlende Werte) auf die Frage „Glauben Sie, dass die Technik alles in allem eher ein Segen oder ein Fluch für die Menschheit ist?" abgebildet. Die möglichen Antwortkategorien sind „eher Segen“ bzw. „eher Fluch“ und „teils/teils“, bzw. „weder noch“ im ersten Messzeitpunkt 1966.

Sofern die Veränderung der Mittelkategorie eine (annähernde) Vergleichbarkeit des Antwortverhaltens erlaubt, können der in Abbildung 1 dargestellten Zeitreihe einige interessante Informationen entnommen werden.

- Zu jedem Messzeitpunkt ruft „Technik“ mehr positive als negative Reaktionen hervor. Abgesehen von 1987 stellen die positiven Urteile absolute Mehrheiten. Dieser Befund spricht klar gegen die oftmals unterstellte Technikfeindlichkeit der Deutschen.

- Nennenswerte Verschiebungen im Antwortverhalten laufen zwischen 1966 und $1976 \mathrm{ab}$, und zwar v. a. zugunsten skeptisch-ambivalenter und zu Lasten affirmativer Haltungen, wohingegen pejorative Assoziationen mit Technik bis zum Ende der Zeitreihe marginal bleiben. Der Wandel zu mehr Skepsis ist 1976 abgeschlossen und nicht mit einer Reihe spektakulärer Technikkatastrophen erklärbar, die erst nach 1976 einsetzten. ${ }^{1}$

Standen die Technikeinstellungen 1966 noch unter dem Eindruck des Wiederaufbaus und nachfolgenden Wirtschaftswunders, weshalb Technik als segensreich empfunden wurde, kam es in der nachfolgenden Dekade zu einem Semantikwandel v. a. von externen Technologien (Abb. 2),
Abb. 1: Globalindikator: Technik - Fluch oder Segen?

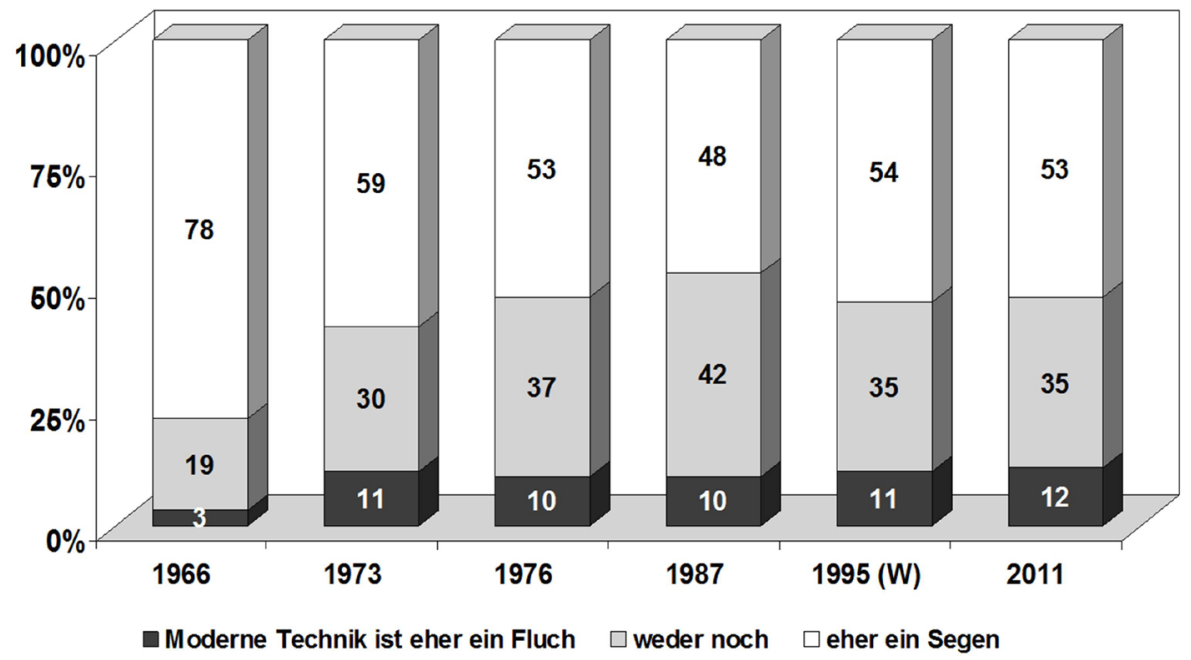

Quelle: Eigene Zusammenstellung auf Basis diverser Umfragen des Instituts für Demoskopie Allensbach, zit. in Renn/Zwick 1997, S. 16; 2011: IfD-Umfrage 10071 (W = Westdeutschland) 
die als Groß- oder Risikotechnik diskutiert wurden. Galten bis dahin Verteilungskämpfe als die zentrale gesellschaftliche Konfliktlinie, setzten postmaterialistisch geprägte Bewegungsanhänger und grüne Parteisympathisanten, die sich als links-ökologischer Arm aus der 68er-Bewegung entwickelten, neuartige Sichtweisen und Forderungen auf die politische Agenda, die sich mit den Attributen ökologisch, partizipatorisch und emanzipatorisch charakterisieren lassen (Zwick 1990, S. 59). Den Zielen, Imperativen und Zumutungen des politisch-administrativen und ökonomischen Systems setzten sie eine lebensweltliche Perspektive entgegen, der sie neben einer kapitalismus- auch eine technokratiekritische Attitüde verliehen. Neben großtechnischen Projekten, allen voran der Kernkraftnutzung, wurde vor allem solche Technik kritisch hinterfragt, deren Externalitäten im Verdacht standen, die Lebenswelten der Menschen zu kolonialisieren (Habermas 1977, S. 61).

Die, ungeachtet aller eingetretenen Technikkatastrophen, hohe Stabilität des Antwortverhaltens seit 1976 legt zum einen die Schlussfolgerung nahe, dass es sich bei unspezifischen Technikeinstellungen weniger um Meinungen handelt, die vom aktuellen Tagesgeschehen abhängen, sondern um relativ stabile Werthaltungen mit vornehmlich positivem oder ambivalentem Gepräge. Zum anderen stellt sich aber die Frage, was Menschen eigentlich assoziieren, wenn sie nach Fluch oder Segen von "Technik“ gefragt werden.

\subsection{Was messen wir, wenn wir Technikeinstellungen messen?}

Das Einstellungsobjekt „Technik“ hat Unschärfen. Die Entwicklung zu zunehmend differenzierten bzw. ambivalenten Technikeinstellungen kann auch darauf zurückgeführt werden, dass „Technik“ als Einstellungsobjekt zu breit und heterogen ist, um einheitliche Bewertungen zu ermöglichen. Technik umfasst Entwicklungen der Alltagstechnik, die zu einer rapiden Technisierung der Ausstattung von Privathaushalten mit Geräten wie Waschmaschinen, Telefonen etc. geführt hat (Zapf et al. 1986), zu medizinischen Hilfsmitteln aber auch zu Kern- kraftwerken und Industrieanlagen. Es war daher folgerichtig, dass „Technik als Globalindikator" an Bedeutung verliert. An die Stelle von Skalen, die das Verhältnis zur Technik im Allgemeinen thematisieren, treten zunehmend Untersuchungen, die sich mit konkreten Technologien beschäftigen, etwa der Gentechnik oder der Energietechnik. Selbst hier ist zu beobachten, dass die Komplexität und Dynamik technischer Entwicklungen eine permanente Herausforderung für die theoretische Konzeptionalisierung des Einstellungsobjekts darstellt, um die gesellschaftliche Diskussion über neue Technologien überhaupt abbilden zu können. Am Beispiel der Gentechnik, einer der Technologien, deren gesellschaftliche Reaktionen am besten untersucht sind, lässt sich zeigen, dass Einstellungen erheblich von ihrer Kontextualisierung beeinflusst sind: Medikamentenentwicklung mittels Gentechnik und das Klonen von Menschen gehören beide zu medizinischen Anwendungen der Gentechnik, sowohl gesellschaftliche Debatten als auch die Einstellungen zu diesen Anwendungen unterscheiden sich jedoch grundlegend (Kap. 4.1).

\subsection{Technikbereiche und ihre Akzeptabilität - und der Mangel an aktuellen Surveydaten für Deutschland}

Als einen ersten, groben Differenzierungsansatz schlägt Renn (zuerst 1986, S. 45) die Unterscheidung dreier Technikbereiche vor: erstens Produkt-, Alltags- und Freizeittechnik, zweitens Technik im Arbeitsleben und drittens externe Groß- und Risikotechnik (Abb. 2):

Die beiden zuerst genannten Technikbereiche erweisen sich hinsichtlich der Akzeptanzfrage in Deutschland als unproblematisch. Bei externer Technik ist hierzulande wie international mit größeren Vorbehalten, teilweise auch Protesten und Akzeptanzverweigerung zu rechnen. Dabei kann jedoch fallweise starke Heterogenität in der Bewertung und Akzeptabilität erwartet werden, die bis in einzelne technologische Anwendungsfelder hineinreicht bzw. von Kontexten und Arrangements abhängt, in die technische Projekte eingebettet sind. 
Abb. 2: Technikbereiche und Parameter ihrer Akzeptanz

\begin{tabular}{|l|l|l|l|}
\hline Technik & Allokationsverfahren & Akzeptanztest & \multicolumn{1}{|c|}{ Konfliktthemen } \\
\hline $\begin{array}{l}\text { Produkt-, Alltags- } \\
\text { und Freizeittechnik }\end{array}$ & Markt & Nachfrage, Kauf & Qualität, Haftung \\
\hline $\begin{array}{l}\text { Technik im } \\
\text { Arbeitsleben }\end{array}$ & Betrieb & Aktive Nutzung & $\begin{array}{l}\text { Mitbestimmung, } \\
\text { Anpassungsgeschwindigkeit, } \\
\text { Qualifikation }\end{array}$ \\
\hline $\begin{array}{l}\text { Externe (Groß-) } \\
\text { Technik }\end{array}$ & Politik & $\begin{array}{l}\text { Politische Partizipation } \\
\text { a) konventionelle } \\
\text { (Abstimmungen) } \\
\text { b) unkonventionelle } \\
\text { (Protestverhalten) }\end{array}$ & $\begin{array}{l}\text { Interessen, Rechte, } \\
\text { Zuständigkeiten, } \\
\text { Legitimität vs. Legalität } \\
\text { Verzerrung der organisierten } \\
\text { Interessen }\end{array}$ \\
\hline
\end{tabular}

Quelle: Renn/Zwick 1997, S. 24

\section{Empirische Befunde}

Dies empirisch zu belegen, fällt allerdings immer schwerer, denn spezielle Techniksurveys, die es erlaubten, ein breiteres Spektrum an Technologien einschätzen zu lassen und die Ursachen für differentielle Technikbewertung differenziert zu analysieren, haben in Deutschland zur Jahrtausendwende ein vorläufiges Ende gefunden. Der Gesis-Datenbestandskatalog (Gesis 2015) weist für Deutschland den letzten Survey zur allgemeinen Technikwahrnehmung für das Frühjahr 1997 aus (Hennen 1997; INIFES/TAB 1998)².

Der Mangel an nationalen Surveydaten wird nur teilweise durch Eurobarometeruntersuchungen ausgeglichen, die in größeren zeitlichen Abständen unterschiedliche Technologien zum Gegenstand haben. Eurobarometerstudien ermöglichen zwar europaweite Vergleiche der Technikeinstellungen. Wann und zu welchen Themen Eurobarometerstudien durchgeführt werden, wird nach politischen, nicht nach wissenschaftlichen Kriterien entschieden - und die Qualität der Erhebungen ist durchaus heterogen. Mitunter scheint das Interesse an der Einschätzung und Beurteilung von Maßnahmen größer zu sein als das Interesse an einer sozialwissenschaftlich präzisen Erfassung und der theoretischen und empirischen Klärung der untersuchten Sachverhalte. Dies ist aus der Perspektive einer politischen Institution durchaus nachvollziehbar, macht aber ein strukturelles Defizit der Forschung zu Technikeinstellungen deutlich. Dennoch geben EurobarometerUntersuchungen - in unterschiedlichem Ausmaß - Informationen, die auf andere Art nicht zu erreichen sind, v. a. die internationale Vergleichbarkeit. Bei einigen Themen sind sogar Zeitreihen verfüg- bar, aber, wie bereits erwähnt, bei vielen Themen fehlen ansonsten jedwede Daten.

In den Eurobarometer-Untersuchungen zur Biotechnologie, die seit 1996, anders als andere Studien, von einem internationalen Expertenteam aus Sozialwissenschaftlern entwickelt wurden, finden sich auch allgemeine Fragen zu Technikeinstellungen. In der Eurobarometer-Befragung 73.1 aus dem Jahr 2010 (EC 2010; Gaskell et al. 2010), der bislang letzten Befragung in dieser Serie, wurden als Proxi für einen Globalindikator zu Technikeinstellungen insgesamt acht Technologien (Abb. 3) zur Einschätzung vorgelegt, mit der Frage, ob man meine, „dass diese Technologien auf unser Leben in den nächsten 20 Jahren einen positiven, einen negativen oder keinen Einfluss haben werden" (Gaskell et al. 2010, S. 132, eigene Übersetzung). Da diese Fragen für einige der untersuchten Technologien seit 1991 regelmäßig gestellt werden, liegen hier Zeitreiheninformationen vor. Da es sich bei diesem Eurobarometer um eine Spezialstudie zu Einstellungen gegenüber der Gentechnik handelt, fehlen vertiefende Fragen zu den übrigen sieben Technologien ebenso wie Prädiktoren, die diesbezügliche Einstellungen aufklären könnten. Konstruiert man aus diesen Fragen einen Summenindex, bei dem aufgetragen wird, wie viele der untersuchten Technologien mit positiven Erwartungen und wie viele von diesen acht mit negativen Erwartungen assoziiert werden, kommt man im EU-Durchschnitt auf 4,9 positiv und 1,1 negativ bewertete Technologien (Gaskell et al. 2010, S. 15). Positive Erwartungen an Technologien überwiegen also deutlich. Die deutsche Öffentlichkeit ist etwas kritischer. Hier werden durchschnittlich 4,7 Technologien positiv und 1,4 Technologien negativ bewertet. Überdurchschnittlich positive Erwartungen finden sich vor allem in Skandinavien, während die Öffentlichkeit in Österreich und der Schweiz besonders skeptisch ist. 
Abb. 3: Bewertung unterschiedlicher Technologien in Deutschland und in der EU 2010

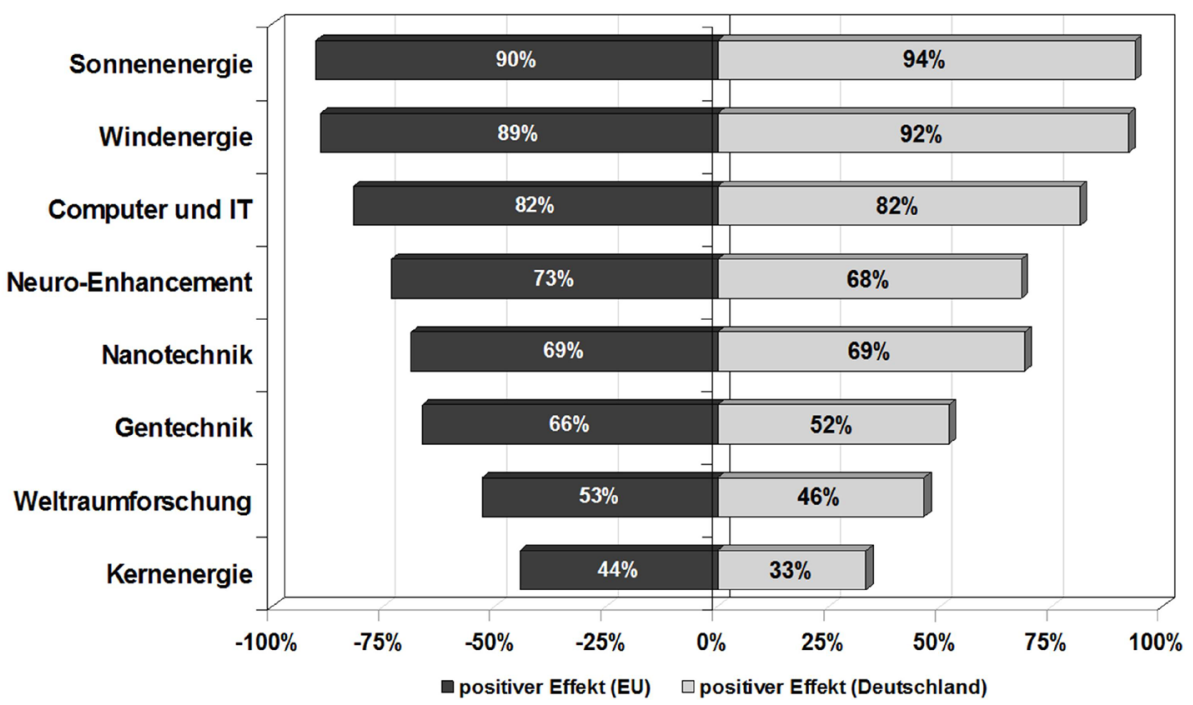

Quelle: Eigene Zusammenstellung auf Basis des Eurobarometer 73.1 (Gaskell et al. 2010, S. 132f.) ohne fehlende Werte. Frage: „,... wird diese Technik eine positive, eine negative oder keine Wirkung auf unser Leben in den nächsten 20 Jahren haben?"

Die Eurobarometer-Daten lassen für Deutschland ein scharfes Profil in der Technikbewertung erkennen.

Wie Abbildung 3 zeigt, belegen die regenerativen Energien die Spitzenpositionen, gefolgt von Computer und IT. Letzteres dürfte im Wesentlichen auf die informationstechnologische Revolution der vergangenen Dekade zurückzuführen sein, mit breiter Marktdurchdringung von IT-Produkten und ihrer beinahe ubiquitären Nutzung in Alltag und Berufsleben. Im Mittelfeld liegen Nanotechnik und Neuro-Enhancement, zwei neue Technologien mit relativ geringem Bekanntheitsgrad und dementsprechend hohen Anteilen von ,weiß nicht"-Antworten (37\% bei Nanotechnik und $18 \%$ bei Neuro-Enhancement). ${ }^{3}$ Es folgen die Gentechnik, die relativ polar wahrgenommen wird und nur knapp mehr positive als negative Erwartungen weckt, und die Weltraumforschung. Die Kernenergie bildet das Schlusslicht mit bei weitem mehr Ablehnung als Zustimmung.

Mit Blick auf das Meinungsklima in Deutschland im EU-Vergleich gelangen Weyer und sein Team auf gleicher Datengrundlage zu der Einschätzung: „Die Hal- tung der Deutschen ... [ist] keineswegs technikfeindlich, in Relation zum EU27-Durchschnitt aber durchaus nüchterner, differenzierter und teilweise skeptischer" (Weyer et al. 2012, S. 327). Vergleicht man die an der Eurobarometerstudie beteiligten Länder, wird ein erhebliches $\mathrm{Maß}$ an Heterogenität bei der Technikbewertung sichtbar. Seitens der Energietechnik schneiden die Deutschen 2010 bei den regenerativen Energien Wind und Sonne etwas besser, bei der Kernkraftnutzung deutlich schlechter ab als der EU-Durchschnitt, worin sich ein Jahr vor der Nuklearhavarie von Fukushima Zustimmung zur späteren Energiewende andeutet. Bei Computern, IT und der Nanotechnik liegt Deutschland im europäischen Durchschnitt, mit bemerkenswert hohen Anteilen fehlender Werte bei letzterer in Deutschland (37\%) und Europa (40\%). Beim Neuro-Enhancement finden wir in Deutschland etwas unterdurchschnittliche Zustimmungsraten. Gleichfalls schlechter als im EU-Durchschnitt wird hierzulande die Weltraumforschung bewertet. Vergleichsweise besonders schlecht schneidet die Gentechnik im Urteil der deutschen Öffentlichkeit ab, wobei sich auch diesbezüglich $18 \%$ der Deutschen und $20 \%$ der Europäer kein Urteil erlauben wollen.

Eine andere Frage ist, welche Erwartungen und Bedenken Menschen haben, wenn sie gebeten werden, Technik zu beurteilen. In dem Survey, der der Studie von Zwick/Renn (1998) zugrunde liegt, konnten die Befragten zu vier Technologien jeweils bis zu drei Argumente benennen, die aus ihrer Sicht für oder gegen eine Technik sprechen. Die Antworten geben einen Eindruck davon, welch heterogene Kriterien Menschen anlegen, wenn sie Technik bewerten sollen: Für Multi- 
media spricht vor allem persönlicher Nutzen, die Bedenken werden vorrangig durch die Unterminierung persönlicher Kontakte und Probleme der Datensicherheit gespeist. Gegen Industrieroboter spricht ein einziger Einwand, nämlich der befürchtete Abbau von Arbeitsplätzen. Positiv schlagen Arbeitserleichterungen und Effizienzbzw. Wettbewerbsvorteile zu Buche. Gegen die Kernenergie werden vor allem wahrgenommene Gefahren und in zweiter Linie Entsorgungsprobleme ins Feld geführt. Auf der Habenseite wird vermerkt, dass sie vorübergehend noch für die Stromversorgung benötigt werde. Ganz anders bei der Gentechnik. Gegen sie werden in dieser Studie weder Technikversagen noch technische Risiken vorgebracht, sondern vor allem Missbrauchsverdacht. Das ist deshalb interessant, weil in diesem Fall nicht „Technik an sich“ zum Gegenstand von Kritik wird. Ihre mehrheitliche Ablehnung vollzieht sich vielmehr vor dem Kontext ihrer gesellschaftlichen Anwendung, der kein hinreichendes Vertrauen entgegengebracht wird. Vorteile dieser Technik werden vorrangig in medizinischen Nutzenpotenzialen erkannt (Zwick/ Renn 1998, S. 19ff.).

\section{Schlussfolgerungen}

Die dargestellten empirischen Befunde legen mehrere Schlussfolgerungen nahe: Sie bestätigen, dass die Deutschen Technik sehr unterschiedlich und überwiegend positiv aufnehmen. Von einer pauschalen Technikferne oder -feindlichkeit kann keine Rede sein.

Neben der Produkttechnik schneiden in der Meinung der Bundesbürger vor allem die regenerativen Energien hervorragend $a b$ - hier finden sich die Deutschen auch europaweit in der Spitzengruppe. Aber auch medizintechnische Anwendungen und in jüngster Zeit die IT-Technik finden großen Anklang. Durch besondere Skepsis zeichnen sich die Deutschen im europäischen Vergleich gegenüber der Kern- und Gentechnik aus; in beiden Fällen sind Technikfelder betroffen, die als externe Technik wahrgenommen werden.

Daraus lassen sich Anforderungen und Kriterien benennen, die über das Maß an Akzeptabilität externer Technik in der Öffentlichkeit entscheiden: Umweltverträglichkeit, Sozialver- träglichkeit, hohe Nutzen- bei geringen Risikopotenzialen, ethische Unbedenklichkeit und, wie wir noch sehen werden, ausreichende politische Legitimierung bei Produktion und Implementation. Ferner weisen Zwick/Renn darauf hin, dass das Vertrauen, das die Öffentlichkeit den verantwortlichen Institutionen, Politik und Betreibern entgegenbringt, wenn es um die Regulierung und Kontrolle groß- und risikotechnischer Anlagen geht, eine wichtige Moderatorvariable darstellt, die maßgeblich über Akzeptanz oder Ablehnung entscheiden kann (Zwick/Renn 2002, S. 46ff.). Vertrauen, verstanden als wahrgenommene Performanz der verantwortlichen Institutionen bei der dauerhaften, zuverlässigen Kommunikation, Regulierung und Kontrolle von Technik und ihren Risiken, verstärkt die Nutzen- und schwächt die Risikowahrnehmung von technischen Projekten ab (Siegrist 2001, S. 24). Allerdings diagnostizieren Zwick/Renn (2002, S. 25) empirisch ein sehr geringes Zutrauen der Öffentlichkeit in die institutionelle Performanz von Politik und Betreibern technischer Anlagen. In einer älteren Studie diagnostizieren sie eine klaffende Glaubwürdigkeitslücke, die sich zwischen Verantwortlichkeit einerseits und Ehrlichkeit, Zuverlässigkeit und Transparenz der Risikokommunikation andererseits auftut (Zwick/Renn 1998, S. 47).

Am Beispiel der Wahrnehmung der Gentechnik zeigt sich, dass hinsichtlich der Technikbewertung weiterer Differenzierungsbedarf besteht. Sie folgt je nach Anwendungsfeld unterschiedlichen Mustern: Manche Anwendungsfelder stoßen auf konsequente Ablehnung, während medizinische und pharmazeutische Anwendungen hohe Nutzenerwartungen und ebensolche Akzeptanz genießen. Im folgenden Abschnitt werden wir diesem Phänomen weiter nachgehen. Weiterer Differenzierungsbedarf wird erkennbar, wenn es, vor allem bei großtechnischen und Infrastrukturprojekten um Ansprüche nach Bürgerbeteiligung geht, und damit verbunden um die Frage nach adäquaten institutionellen Arrangements bei Implementation und Betrieb der entsprechenden Anlagen, ein Thema, das wir im Rahmen dieses Beitrags aber nur am Rande streifen können. 


\section{Einstellungen gegenüber konkreten Technologien und spezifischen Anwendungsfeldern}

\subsection{Differentielle Technikakzeptanz je nach Anwendungsfeld am Beispiel der Gentechnik}

Gentechnik gehört zu den am stärksten kontrovers diskutierten Technologien der vergangenen Jahrzehnte (Torgersen et al. 2002). Bereits im Jahr 1991 wurde die erste Eurobarometer-Untersuchung zur Wahrnehmung der Gentechnik durchgeführt, der bis zum Eurobarometer 73.1 (EC 2010) insgesamt sechs weitere Surveys folgten. Vor allem die Befragungen seit 1996 erhielten mit der Einberufung einer Wissenschaftlergruppe zur Entwicklung der Erhebungsinstrumente und zur Auswertung einen stärker wissenschaftlichen Charakter als andere Eurobarometer-Studien. Parallel zu den Surveys von 1996 und 1999 wurden darüber hinaus sowohl Analysen der Medienberichterstattung in den beteiligten Ländern als auch Analysen des politischen Diskurses über Gentechnik durchgeführt (Gaskell et al. 1997; Durant et al. 1998; Gaskell/ Bauer 2001; Bauer/Gaskell 2002; Gaskell/Bauer 2006; Gaskell et al. 2007; Gaskell et al. 2010). In Deutschland hat seit Mitte der 1990er Jahre ein von der Akademie für Technikfolgenabschätzung in Baden-Württemberg koordiniertes Projekt systematisch die Einstellungen zur Gentechnik untersucht (Hampel/Renn 1999).

Sowohl die internationalen als auch die deutsche Studie ergaben, dass die Gentechnik kein einheitliches Konstrukt ist, dass vielmehr die verschiedenen Anwendungen sehr unterschiedlich wahrgenommen und bewertet werden. Die unterstellte generelle Ablehnung der Gentechnik konnte empirisch nicht bestätigt werden. Außerdem fällt die zeitliche Konstanz der Einstellungen zu verschiedenen Anwendungsfeldern auf.

Bereits die Studien der 1990er Jahre haben gezeigt, dass medizinische Anwendungen der Gentechnik gemeinhin akzeptiert werden, während gegenüber landwirtschaftlichen Anwendungen starke Vorbehalte bestehen (Hampel/Renn 1999). Letzteres schließt Vorbehalte gegen den Gentechnikeinsatz in der Lebensmittelproduktion sowie die gentechnische Veränderung von Tieren ein, die auch in aktuellen Studien zu be- obachten sind, aber infolge der wissenschaftlichen Dynamik der Gentechnik weiter differenziert werden müssen. ${ }^{4}$

An der verbreiteten Ablehnung gentechnisch veränderter Lebensmittel hat sich auch in den aktuellen Studien nichts Grundlegendes verändert. Auch 2010 werden gentechnisch veränderte Lebensmittel mehrheitlich abgelehnt.

Eine Ursache für die geringe Beliebtheit gentechnisch veränderter Nahrungsmittel sind vermutete Risiken; die überwiegende Mehrheit betrachtet die Grüne Gentechnik als eine Risikotechnologie ${ }^{5}$, wobei vor allem zukünftige Risiken befürchtet werden, wovon fast drei Viertel der Deutschen (72\%) überzeug sind.

Die Annahme, dass die Risikowahrnehmung ausschlaggebend für die geringe Unterstützung gentechnisch veränderter Lebensmittel ist, hat sich jedoch als falsch herausgestellt. Gaskell et al. (2004) zufolge ist nicht die Risikowahrnehmung maßgeblich für die Ablehnung gentechnisch veränderter Nahrungsmittel, sondern wahrgenommene Nutzenpotenziale: Ohne Nutzenwahrnehmung keine Akzeptanz! (vgl. auch Hampel/Pfenning 1999, S. 46; Zwick/Renn 2008, S. 83) Hier ist die Achillesferse der Grünen Gentechnik, da sowohl der individuelle wie auch der wirtschaftliche Nutzen nur sehr gering eingeschätzt werden: Nur ein Drittel der Befragten erachtet gentechnisch veränderte Lebensmittel als für die nationale Wirtschaft nützlich.

Andere Entwicklungen der Gentechnik werden dagegen deutlich positiver wahrgenommen. Ein damals überraschendes Ergebnis der deutschen Biotech-Befragung von 1997 war, dass beispielsweise pharmazeutische Anwendungen und Produkte der Gentechnik auf große Zustimmung stießen (Hampel/Renn 1999). Dieser Befund hat sich empirisch erhärtet.

Eine der am intensivsten geführten Diskussionen im Bereich der Gentechnik war die Diskussion um die Forschung mit embryonalen menschlichen Stammzellen (Cheveigné et al. 2006). In den Eurobarometer-Untersuchungen von 2005 und 2010 wurde differenziert nach der Einstellung zu embryonalen und adulten Stammzellen gefragt. Überraschenderweise waren die Unterschiede in der Wahrnehmung der ethisch unbedenklichen Forschung mit adulten Stamm- 
zellen und der ethisch kontrovers diskutierten Forschung mit embryonalen Stammzellen nur sehr gering (Gaskell et al. 2007; Gaskell et al. 2010). Medizinische Anwendungen der Gentechnik werden aber nicht per se positiv bewertet, wie das Beispiel Gentherapie zeigt (Hampel 2011).

Von Entwickler- und Produzentenseite wird immer wieder mangelnder technischer Kenntnisstand in der Öffentlichkeit beklagt, verbunden mit der Erwartung, dass hohes technikbezogenes Sachwissen die Technikakzeptanz verbessere. Diese Hoffnung ist jedoch unbegründet. Empirisch zeigt sich nämlich, dass sich beispielsweise unter den am stärksten über die Gentechnik Informierten zwar die eifrigsten Befürworter, aber auch die entschiedensten Gegner befinden, die ihre Ablehnung mit Sachwissen und Argumenten untermauern. So gesehen trägt steigendes Wissen eher zu einer Polarisierung der Technikbewertung bei (Hampel/Pfenning 1999; Hampel 2004, S. 48.)

\subsection{Für wen ist Großtechnik akzeptabel und warum?}

Anders als noch vor Dekaden, laufen Versuche, über soziodemografische Merkmale technikaufgeschlossene Menschen von Technikgegnern zu unterscheiden, ins Leere. Gesellschaftliche Modernisierungsprozesse haben dazu geführt, dass sich soziale Großgruppen, die über soziodemografische Variablen erfasst werden konnten und in enger Verbindung mit gesellschaftlichen Organisationen standen - man denke etwa an die „Arbeiterschaft“ und ihre Assoziation mit Sozialdemokratie und Gewerkschaften -, aufgelöst und ihre Bindungskraft weitestgehend verloren haben. Allenfalls Männer, junge und hoch gebildete Personen erweisen sich Technik gegenüber ein wenig aufgeschlossener als der Durchschnitt, aber diese Effekte sind, wie das Eurobarometer von 2010 zeigt, gering.

An die Stelle der prägenden Kraft von gesellschaftlichen Großgruppen sind differentielle soziokulturelle Milieus getreten, mit je unterschiedlichen Weltbildern und Lebensstilen ihrer Protagonisten, durch die auch technikbezogene Einstellungen und Werthaltungen geprägt sind. Dies trifft, wie Zwick/Renn empirisch ermitteln konnten (1998, S. 57; Zwick/Renn 2002, S. 64), vor allem auf Personen zu, die sich entweder als „technokra- tisch-liberale Aufstiegsorientierte" oder aber als „modernisierungsfeindliche, kulturpessimistische Alternative“" (Zwick/Renn 1998, S. 57ff.) charakterisieren lassen. Letztere erweisen sich vor allem Groß- und Risikotechniken gegenüber erheblich ablehnender als ,Technokraten“. 6

Es muss davon ausgegangen werden, dass für die differentielle Wahrnehmung von Technik in der Gesellschaft, nicht nur „Technik an sich“, ihre Eigenschaften und Folgen maßgeblich sind, sondern auch ihre Symbolkraft. Technik vermag Stolz, Hoffnungen, Fortschrittsoptimismus, aber auch Enttäuschungen, Bedenken oder Ängste hervorrufen. Mit Technik wird in die Natur eingegriffen und vertrauter Lebensraum umgestaltet. Dabei markiert Technik ,eine semantische Kluft zwischen ,künstlich' und ,natürlich', [die] in Deutschland besonders stark aufgeladen ist ... Je näher eine Technik dem Pol ,Natur' zugeordnet wird (etwa Solarkollektoren), umso emphatischer wird diese Technik in der Wahrnehmung der Bevölkerung bewertet" (acatech 2011, S. 15) und vice versa.

Protagonisten von kontrovers diskutierten Technologien pflegen Widerstände der Öffentlichkeit gelegentlich als emotional und irrational abzutun, verbunden mit der Hoffnung auf eine sachlich-rationale Betrachtungsweise. Dies verstellt den Blick darauf, dass kritische Reaktionen auf Technik zumeist einer rationalen Bilanzierung von Nutzen- und Schadenspotenzialen sowie deren gesellschaftlicher Verteilung folgt, aber auch auf wahrgenommene Regulierungsdefizite und fehlendes Vertrauen in die Akteure der Technisierung zurückgeführt werden kann. Diese Gesichtspunkte sind sehr gut mit der Theorie rationaler Akteure (Fishbein/Ajzen 1975, Ajzen/ Fishbein 1980, s. a. Mayerl 2009, S. 59-76) vereinbar. Hinter dem Vorwurf, die Ablehnung neuer Technologien sei emotional, steht daher mitunter nur die Kritik daran, dass die Öffentlichkeit andere Kriterien für die rationale Bewertung einer Technik ansetzt als Experten - ein Phänomen, das beispielsweise aus der Risikowahrnehmungsforschung seit langem bekannt ist (Slovic 1987).

Darüber hinaus gibt es emotionale Aspekte, die individuelle Reaktionen auf neue Technologien beeinflussen. Technikbezogene Emotionen können zum einen beispielsweise akut durch Besitzerstolz, Technikversagen oder -katastrophen 
hervorgerufen werden, wobei die Abgrenzung zu kognitiven Reaktionen schwer fällt. Zum anderen können sie aber auch dann noch persistieren, wenn das prägende Ereignis längst in Vergessenheit geraten ist. So gesehen repräsentieren sie eine halb- oder unbewusste Aufschichtung von Ereignissen, die als positive, optimistische Emotionen gegenüber Technik oder aber mit Unbehagen und Ängsten behaftet, langfristig mental abgelegt werden. Als ,sedimentiertes historisches Gedächtnis“ reduzieren sie Komplexität und verleihen den Akteuren Orientierungssicherheit. In ähnlicher Weise können technikbezogene Emotionen aber auch durch wahrgenommenes Ver- oder Misstrauen gegenüber Produzenten oder verantwortlichen Regulatoren, über wahrgenommene Verantwortungsübernahme und Zuverlässigkeit ${ }^{7}$, aber auch durch Skandale und Kommunikationsversagen dauerhaft verinnerlicht werden und das Image einer Technologie ebenso langfristig prägen wie das von verantwortlichen Akteuren und Institutionen.

In einer Studie ließen Zwick/Renn sechs Technologien auf einer Ratingskala zwischen Angst auf der einen und Begeisterung auf der anderen Seite einschätzen (1998, S. 35). Dabei ergaben sich dramatische Unterschiede: Sonnenenergie rief nur bei $1 \%$ der Befragten Angst hervor, bei Handys waren es $4 \%$ und bei Multimedia $6 \%$. Industrieroboter machten $16 \%$ der Befragten Angst, während die Befragten gegenüber Gentechnik (51\%) und Kernenergie (60\%) mehrheitlich Ängste äußerten (1998, S. 32). Vor allem auf die zuletzt genannten Technologien reagierten Frauen erheblich häufiger mit Angst als Männer. ${ }^{8}$ In dieser vergleichenden Studie erwiesen sich technikbezogene Emotionen als starke Prädiktoren für die Frage nach der Akzeptabilität einer Technik.

\subsection{Die soziale Kontingenz von Technikeinstellungen am Beispiel der Windenergie}

Negative Einstellungen zu neuen Technologien führen nicht automatisch zu Technikkonflikten. Technikkonflikte entstehen in einem komplexen Zusammenwirken von Öffentlichkeit, gesellschaftlichen Akteuren wie NGOs, Medien und politischen Rahmenbedingungen (Renn 1998; Hampel/Torgersen 2010; Torgersen/Hampel
2012). Über das Ausmaß, in welchem Technik akzeptiert wird, entscheidet nicht nur die Wahrnehmung und Bewertung von technischen Artefakten oder Anlagen und ihre Eigenschaften, sondern im Fall externer Technik maßgeblich, auf welche Weise Technik eingeführt wird, inwieweit Betroffene an Entscheidungen beteiligt werden und welche institutionellen Arrangements des Anlagenbetriebs gewählt werden. Exemplarisch lässt sich dies an zwei Windkraftprojekten in den Schwarzwaldgemeinden Freiamt und Simmersdorf veranschaulichen, die von Schmid/Zimmer (2012) eingehend analysiert wurden.

In beiden Fällen geht es um die gleiche Technik, die soziale Einbettung dieser Technik unterscheidet sich aber grundlegend: In Simmersfeld wurde der Windpark durch einen Energiekonzern initiiert. Die meisten von Schmid und Zimmer befragten Bürger erfuhren ,aus der Zeitung von dem Projekt... Die Kommunikationsprozesse und die Beteiligung ... [liefen] innerhalb des formalen Rahmens ab... Informationsveranstaltungen [wurden] erst als Reaktion auf Widerstände insbesondere seitens der Bürgerinitiative" angeboten (Schmid/Zimmer 2012, S. 57). In den durchgeführten Interviews wurde über die verspätete, unzureichende Informationsstrategie geklagt, aber auch, dass vor Ort keine Institution eingerichtet worden sei, an die sich betroffene Anwohner mit Fragen oder Bedenken hätten wenden können; insgesamt berichten die Autoren über ein von Anfang an gestörtes Vertrauensverhältnis gegenüber den Planern und dem ortsfremden Betreiber (Schmid/Zimmer 2012, S. 58).

Die Initiative der Windenergienutzung in Freiamt ging 1997 von Bürgern der Gemeinde aus, die in Eigenregie und „mit viel Enthusiasmus" einen knapp 50 Meter hohen Mast mit einer Windmessanlage errichteten, der, wie es auf der Internetpräsentation des Vereins zur Förderung der Windenergie in Freiamt ${ }^{9}$ heißt, ,sogar dem Orkan ,Lothar' im Dezember 1999 getrotzt" hat und über zwei Jahre hinweg ein erhebliches Windaufkommen registrierte. Vor Ort wurde, wie auch Schmid und Zimmer bei ihren Recherchen feststellten, von Beginn an viel Wert auf Information, Transparenz und Beteiligungsmöglichkeiten bei der Planung gelegt (Schmid/Zimmer 2012, S. 56). Die Entscheidung fiel zugunsten 
eines Bürgerwindparks mit Teilhabern, die sich laut Internetauftritt des Vereins zu rund einem Drittel aus Bürgern der Gemeinde rekrutierten.

Gegenüber fremd betriebenen haben Bürgerwindkraftanlagen für die Anwohner mehrere Vorteile: Der ökonomische Nutzen verbleibt vor Ort, die Verteilung von Nutzen und Lasten, die sich bei externer Technik oftmals als problematisch erweist, wird als gerecht empfunden. Aber auch die Informationswege, Entscheidungsprozesse und Beteiligungschancen können effektiv gestaltet werden. All dies schlägt sich auf die Rolle und das Selbstverständnis der Menschen nieder. Als Beteiligte an der Projektplanung und als Teilhaber sind wesentliche Bedingungen für eine positive Identifikation mit dem Erreichten erfüllt: „In Freiamt waren die Bürgerinnen und Bürger stolz darauf, dass sie den Bürgerwindpark auf die Beine gestellt hatten" (Schmid/Zimmer 2012, S. 57), wohingegen in Simmersfeld allenfalls von einer Tolerierung gesprochen werden kann, nicht aber von ,aktiver Zustimmung“, geschweige denn von einer „Identifizierung der Simmersfelder mit dem Windpark" (Schmid/Zimmer 2012, S. 60). Die Wahrnehmung der Windkraftanlage wird von weit reichender Unzufriedenheit getragen; beklagt werden neben unzureichenden Informations- und Beteiligungsmöglichkeiten auch die unzureichende Qualität des Planungsprozesses. (Schmid/Zimmer 2012, S. 56).

Dieses Beispiel zeigt, dass und wie frühzeitige, faire, transparente und offene Informationsund Planungsprozesse sowie das gewählte Arrangement, unter dem Anlagen betrieben werden, wie ferner Nutzen und Lasten verteilt werden, Zufriedenheit und Selbstverständnis der involvierten Bürgerinnen und Bürger beeinflussen können. Neben einer positiven Identifizierung mit dem Projekt kann es zu einem positiven Framing vermeintlicher oder tatsächlicher Nachteile kommen. Ist man bereit, die aufgezählten Eigenschaften gemeinsam unter Nutzen zu subsumieren, scheint es gerechtfertigt, wie unlängst am Beispiel des Mobilfunks und seiner Risiken erlebt, zu konstatieren: Wahrgenommener Nutzen kann Risiken kannibalisieren.

Zusammenfassend belegen die beiden Fallstudien, dass eine auf die Wahrnehmung von Technik abzielende Analyse ihrer Akzeptabilität zu kurz greift. Vor allem bei externer und Infra- strukturtechnik kommt es maßgeblich darauf an, ob die betroffenen Bürger adäquat informiert und an den Entscheidungen beteiligt werden. Großund infrastrukturtechnische Projekte diskriminieren typischerweise zwischen vielen anonymen Nutznießern und wenigen, die ein Übermaß an Lasten zu tragen haben. Die beiden Windkraftprojekte in Freiamt und Simmersfeld legen Zeugnis davon ab, dass Technikakzeptanz - hier allenfalls Tolerierung durch die Anwohner, dort die positive Identifizierung mit dem Projekt - in hohem Ausmaß davon abhängt, dass ein Arrangement gewählt wird, das durch selektive Nutzenaspekte dazu beiträgt, dass die sonst zu erwartenden Ungerechtigkeiten in der Verteilung von Nutzen und Lasten kompensiert werden. Es spricht vieles dafür, dass diese Strategien auch bei anderen, strittigen Infrastrukturmaßnahmen, wie etwa bei den für die Energiewende zu errichtenden Stromtrassen, Energiespeichern etc. modifiziert angewandt werden und die Akzeptanz bei den Betroffenen verbessern können.

Deekeling Arndt Advisors schlussfolgern: „Nach Meinung vieler Fachleute sind Vorhabenträger gut beraten, sich von der Sichtweise zu lösen, dass eine Planung, die auf dem Papier legal ist, automatisch auch in den Augen der übrigen Gesellschaft rechtens ist ... In Beteiligung zu investieren, kann Prozesse unter dem Strich beschleunigen und zu mehr Planungssicherheit verhelfen, lautet eine der zentralen Erkenntnisse dieser Untersuchung. Denn wo Konflikte frühzeitig diskutiert und aus dem Weg geräumt werden, kann Akzeptanz wachsen und erhöhen sich die Chancen für einen Konsens.“ (2012, S. 18f.)

\section{Fazit}

Die Einstellungen der deutschen Öffentlichkeit zu Wissenschaft und Technik haben sich in den vergangenen Jahren stark ausdifferenziert. Verändert hat sich aber auch die wissenschaftliche Herangehensweise. Die noch in den 1990er Jahren gängige Fragestellung, ob die Deutschen technikfeindlich seien, wird in dieser Form nicht mehr gestellt. Zumindest nicht bei denen, die sich wissenschaftlich mit diesen Fragen beschäftigen. $\mathrm{Ob}$ es überhaupt so etwas gibt wie eine „Technikeinstellung“ muss kritisch reflektiert werden, wenn man betrachtet, 
wie unterschiedlich konkrete Technologien oder unterschiedliche Anwendungen von Technologien bewertet werden.

Die Forschung zum Thema Technikeinstellungen hat ein zunehmend differenziertes Bild ergeben. Zwar sind individuelle Charakteristi$\mathrm{ka}$, die zu unterschiedlicher Offenheit in Bezug auf Technik führen, durchaus noch im Fokus der Forschung. An Bedeutung gewonnen haben aber v. a. Analysen, die Technik als soziotechnisches System (Ropohl 1988) betrachten und die erwarteten Effekte des Einsatzes von Technik wie auch die Frage des Vertrauens in die Akteure, die Technisierungsprojekte betreiben, untersuchen ${ }^{10}$. Für die Forschung zum Thema Technikeinstellungen ergibt sich daraus die Notwendigkeit, statt abstrakter Globalindikatoren präzise Anwendungsfelder zu untersuchen und dabei die zentralen sozialwissenschaftlichen Prädiktoren einzubeziehen, anhand welcher sich Technikeinstellungen adäquat erklären und interpretieren lassen. Multi-Methodenstudien, bei denen quantitative Verfahren der Surveyforschung durch qualitative Methoden ergänzt werden, erscheinen in besonderem Maße wünschenswert.

Die Ergebnisse der Forschung zu Technikeinstellungen haben erheblichen Einfluss auf unser Verständnis der Probleme und Möglichkeiten der Technikkommunikation. Die Ablehnung von konkreten Technologien bzw. deren Anwendung - nichts anderes ist zu beobachten - hat konkret benennbare Ursachen, stellt jedoch keine generelle Technikfeindschaft dar. Auch Technikkonflikte können unterschiedliche Ursachen haben. In der Literatur wird etwa zwischen Wissens-, Interessens- und Wertkonflikten unterschieden (Bogner/Menz 2010). Kommunikation, die vor diesem Hintergrund daran ansetzt, im Sinn einer Werbesemantik Technikbegeisterung zu induzieren, ist vor diesem Hintergrund unterkomplex.

Kommunikation ist notwendig, aber kein Allheilmittel. Wenn eine Technologie abgelehnt wird, weil sie zu Ergebnissen führt, die negativ bewertet werden oder Entwicklungen befördert, die abgelehnt werden, weil sie etwa eigene Interessen gefährden oder mit eigenen Werten inkompatibel sind, ist Kommunikation nur sehr bedingt geeignet, daran etwas zu ändern. Kommunikation kann aber helfen, Entscheidungen vorzubereiten, die weniger kontrovers sind. Dementsprechend hat sich die Diskussion in der Scientific Community vom Public-Understanding-of-Science-Paradigma hin zu Konzepten wie Upstream Engagement und Responsible Research and Innovation (RRI) entwickelt, die statt der Kommunikation einer fertig entwickelten Technologie Kommunikationsprozesse mit Stakeholdern und der Öffentlichkeit bereits bei der Entwicklung einer Technik vorschlagen (Rowe/Frewer 2005; Schomberg 2011; Owen et al. 2012).

Das frühzeitige Einbeziehen der Öffentlichkeit in Diskussions- und Entscheidungsprozesse, gar ihre Beteiligung am Betrieb technischer Anlagen, kann sich, wie die beiden knapp skizzierten Windkraftprojekte exemplarisch zeigen, sehr günstig auf die Technikbewertung auswirken. In Einzelfällen lässt sich aber nicht vermeiden, dass Technisierungsprojekte gleichwohl kontrovers verlaufen. Technikkonflikte beruhen nicht nur auf unterschiedlichen Vorstellungen, sondern auch auf divergierenden Interessen und disparaten Werthaltungen. Es ist daher unverzichtbar, dass Kommunikation auf Wissen über die Gründe für diese Kontroversen und nicht auf simplifizierenden Annahmen beruht.

\section{Anmerkungen}

1) Beispielsweise die Chemiekatastrophen von Seveso (1976) und Bhopal (1986), die Kernkraftunfälle von Harrisburg (1979), Sellafield (1984) und Tschernobyl (1986), die Chemieunfälle von Sandoz und Ciba (1986), aber auch eine Serie von fatalen Unfällen der zivilen Luftfahrt (alleine 25 Abstürze im Jahr 1976 und 20 1977) und wiederholte Havarien von Öltankern, darunter beispielsweise die Exxon Valdez und Kharg (1989). Als Nebenfolge des industriellen Lebensstils und Technikeinsatzes wurde in Deutschland das Waldsterben ausgerufen; infolge des sauren Regens galten 1984 mehr als $50 \%$ des Deutschen Waldes als krank.

2) Technikeinstellungen werden seither entweder kursorisch im Rahmen von Mehrthemenbefragungen abgehandelt oder aber es werden einzel$n e$ Technologien in speziellen Studien erforscht.

3) In der Graphik sind nur die abgegebenen Urteile ohne Ausfälle enthalten.

4) Ein Überblick über die aktuelle Entwicklung in diesem Bereich findet sich etwa im 3. Gentech- 
nologiebericht der Berlin-Brandenburgischen Akademie der Wissenschaften und der Publikation der zentralen Ergebnisse der Eurobarometer-Befragung 73.1 (Müller-Röber et al. 2015; Gaskell et al. 2010).

5) $\mathrm{Ob}$ gentechnisch veränderte Lebensmittel tatsächlich riskanter sind als herkömmliche Züchtungen ist nicht Gegenstand dieser Untersuchung. Das Thomas-Theorem besagt, dass das, was Menschen als real betrachten, in seinen Auswirkungen real ist.

6) Die Anteile an Befragten mit positiver Haltung zur Gen- oder Kerntechnik differieren zwischen beiden Gruppen um jeweils mehr als 40 Prozentpunkte (Zwick 1998, S. 80; Zwick/Renn 1998, S. 57); technische Details finden sich in Zwick (1998, S. 68ff).

7) Giddens spricht in diesem Zusammenhang von aktivem Vertrauen (Giddens 1995, S. 48f.; Giddens 1996, S. 319).

8) Die Differenzen sind in beiden Fällen etwas größer als 25 Prozentpunkte.

9) http://www.freiamt-windmuehlen.de/ (download 2.9.15)

10) S. bspw. die Eurobarometerbefragungen zum Thema Biotechnologie oder Bauer/Gaskell (1999).

\section{Literatur}

acatech - Deutsche Akademie der Technikwissenschaften, 2011: Akzeptanz von Technik und Infrastrukturen. Anmerkungen zu einem aktuellen gesellschaftlichen Problem. Acatech Bezieht Position Nr. 9, München; http:/www.acatech.de/fileadmin/user_upload/Baumstruktur_nach_Website/Acatech/root/de/Publikationen/ Stellungnahmen/acatech_bezieht_Position_Nr9_Akzeptanz-von-Technik_WEB.pdf (download 22.3.16)

Ajzen, I.; Fishbein, M., 1980: Understanding Attitudes and Predicting Social Behaviour. Englewood Cliffs, NJ

Bauer, M.; Gaskell, G., 1999: Towards a Paradigm for Research on Social Representations. In: Journal for the Theory of Social Behaviour 29/2 (1999), S. 163-186

Bauer, M.; Gaskell, G. (Hg.), 2002: Biotechnology The Making of a Global Controversy. Cambridge

Bogner, A.; Menz, W., 2010: Konfliktlösung durch Dissens? Bioethikkommissionen als Instrument der Bearbeitung von Wertkonflikten. In: Feindt, P.H.; Saretzki, Th. (Hg.): Umwelt- und Technikkonflikte. Wiesbaden, S. 335-353

Cheveigné, S. de; Einsiedel, E.; Hampel, J., 2006: Spare Parts for Human Bodies? In: Gaskell, G.; Bau- er, M. (Hg.): Genomics \& Society: Legal, Ethical \& Social Dimensions. London, S. 28-43

Deekeling Arndt Advisors, 2012: Akzeptanz für Großprojekte, eine Standortbestimmung über Chancen und Grenzen der Bürgerbeteiligung in Deutschland. Düsseldorf

Durant, J.; Bauer, M.; Gaskell, G. (Hg.), 1998: Biotechnology in the Public Sphere. A European Sourcebook. London

EC - Europäische Kommission, 2010: Eurobarometer 73.1. Durchgeführt von TNS Opinion \& Social, Brüssel. Gesis Datenbestandsarchiv, Köln: Datensatz ZA5000 Version 4.0.0, doi:10.4232/1.11428

Fishbein, M.; Ajzen, I., 1975: Belief, Attitude, Intention, and Behaviour. Reading, MA

Gaskell, G.; Allum, N.; Wagner, W. et al., 2004: GM Foods and the Misperception of Risk Perception. In: Risk Analysis 24/1 (2004), S. 185-194

Gaskell, G.; Bauer, M. (Hg.), 2001: Biotechnology 1996-2000: The Years of Controversy. London

Gaskell, G.; Bauer, M. (Hg.), 2006: Genomics \& Society. Legal, Ethical \& Social Dimensions. London

Gaskell, G.; Stares, S.; Allansdottir, A. et al., 2007: Europeans and Biotechnology in 2005: Patterns and Trends. Luxembourg

Gaskell, G.; Stares, S.; Allansdottir, A. et al., 2010: Europeans and Biotechnology in 2010. Luxembourg

Gaskell, G.; Wagner, W.; Hampel, J. et al., 1997: Europe Ambivalent on Biotechnology. In: Nature 387 (1997), S. 845-846

Gesis, 2015: Datenbestandskatalog, Mannheim; https:// dbk.gesis.org/dbksearch/sdesc.asp?search=technikakze ptanz\&field $=$ all $\& \mathrm{db}=\mathrm{d} \&$ sort $=\mathrm{MA}+\mathrm{DESC} \& \max \mathrm{Rec}=1$ $00 \& n f=1 \&$ from $=$ index. asp \&button= Suchen $\&$ groups $=$ on $\&$ product $=$ on $\& 11=10 \&$ tab $=0($ download 27.8 .15$)$

Giddens, A., 1995: Konsequenzen der Moderne. Frankfurt a. M.

Giddens, A., 1996: Risiko, Vertrauen und Reflexivität. In: Beck, U.; Giddens, A.; Lash, S. (Hg.): Reflexive Modernisierung. Frankfurt a. M., S. 316-337

Grunwald, A., 2008: Akzeptanz und Akzeptabilität technikbedingter Risiken. In: Grunwald, A.: Technik und Politikberatung. Frankfurt a. M., S. 339-367

Habermas, J., 1977: Legitimationsprobleme im Spätkapitalismus. Frankfurt a. M.

Hampel, J., 2004: Die Akzeptanz gentechnisch veränderter Lebensmittel. In: Stuttgarter Beiträge zur Risiko- und Nachhaltigkeitsforschung Nr. 3. Stuttgart Hampel, J., 2011: Wahrnehmung und Bewertung der Gentherapie in der deutschen Bevölkerung. In: Fehse, 
B.; Domasch, S. (Hg.): Gentherapie in Deutschland. Eine interdisziplinäre Bestandsaufnahme. Themenband der Interdisziplinären Arbeitsgruppe Gentechnologiebericht der Berlin-Brandenburgischen Akademie der Wissenschaften. Dornburg, S. 227-255

Hampel, J.; Pfenning, U., 1999: Einstellungen zur Gentechnik. In: Hampel, J.; Renn, O. (Hg.): Gentechnik in der Öffentlichkeit. Wahrnehmung und Bewertung einer umstrittenen Technologie. Frankfurt a. M.

Hampel, J.; Renn, O. (Hg.), 1999: Gentechnik in der Öffentlichkeit. Wahrnehmung und Bewertung einer umstrittenen Technologie. Frankfurt a. M.

Hampel, J.; Torgersen, H., 2010: Der Konflikt um die Grüne Gentechnik und seine regulative Rahmung. Frames, Gates und die Veränderung der europäischen Politik zur Grünen Gentechnik. In: Feindt, P.H.; Saretzki, Th. (Hg.): Umwelt- und Technikkonflikte. Wiesbaden, S. 143-163

Hennen, L., 1994: Ist die (deutsche) Öffentlichkeit „technikfeindlich“? Ergebnisse der Meinungs- und der Medienforschung. Erster Sachstandsbericht im Rahmen des Monitoring-Projektes „Technikakzeptanz und Kontroversen über Technik" am TAB. TAB Arbeitsbericht 24. Bonn

Hennen, L., 1997: Monitoring „Technikakzeptanz und Kontroversen über Technik“: Ambivalenz und Widersprüche: Die Einstellung der deutschen Bevölkerung zur Technik. Ergebnisse einer repräsentativen Umfrage des TAB, 2. Sachstandsbericht, 1997. Arbeitsbericht Nr. 54, Bonn

Huber, J., 1989: Technikbilder. Opladen

INIFES - Internationales Institut für empirische Sozialökonomie gGmbH; TAB - Büro für TechnikfolgenAbschätzung beim Deutschen Bundestag, 1998: Die Einstellung der deutschen Bevölkerung zur Technik. GESIS Datenarchiv. Köln

Mayerl, J., 2009: Kognitive Grundlagen sozialen Verhaltens. Framing, Einstellungen und Rationalität. Wiesbaden

Miller, J.D., 2004: Public Understanding Of, and Attitudes Towards, Scientific Research: What We Know and What We Need To Know. In: Public Understanding of Science 13 (2004), S. 273-294

Müller-Röber, B.; Budisa, N., Diekämper, J. et al., 2015: Dritter Gentechnologiebericht. Analyse einer Hochtechnologie. Forschungsberichte der interdisziplinären Arbeitsgruppen der Berlin-Brandenburgischen Akademien der Wissenschaften. Baden-Baden

National Science Board, 2014: Science and Engineering Indicators 2014; http://www.nsf.gov/statistics/ seind14/index.cfm (download 23.11.15)
Owen, R.; Macnaghten, P.; Stilgoe, J., 2012: Responsible Research and Innovation: From Science in Society to Science for Society. In: Science and Public Policy 39 (2012), S. 751-760

Renn, O., 1986: Akzeptanzforschung. Technik in der gesellschaftlichen Auseinandersetzung. In: Chemie in unserer Zeit 20/2 (1986), S. 44-52

Renn, O., 1998: Die Austragung öffentlicher Konflikte um chemische Produkte oder Produktionsverfahren - Eine soziologische Analyse. In: Renn, O.; Hampel, J. (Hg.): Kommunikation und Konflikt. Fallbeispiele aus der Chemie. Würzburg, S. 11-51

Renn, O.; Zwick, M.M., 1997: Risiko- und Technikakzeptanz. Enquete-Kommission „Schutz des Menschen und der Umwelt" des Deutschen Bundestages. Berlin

Ropohl, G., 1988: Zum gesellschaftstheoretischen Verständnis soziotechnischen Handelsn im privaten Bereich. In: Joerges, G. (Hg.): Technik im Alltag. Frankfurt a. M., S. 120-144

Rowe, G.; Frewer, L.J., 2005: A Typology of Public Engagement Mechanisms. In: Science, Technology, and Human Values 30/2 (2005), S. 251-290

Schmid, S.I.; Zimmer, R., 2012: Akzeptanz von Windkraftanlagen in Baden-Württemberg. Unabhängiges Institut für Umweltfragen, UfU-Paper 2/12. Berlin

Schomberg, R. von, 2011: Prospects for Technology Assessment in a Framework of Responsible Research and Innovation. In: Dusseldorp, M.; Beecroft, R. (Hg.): Technikfolgen abschätzen lehren. Wiesbaden, S. 39-61

Siegrist, M., 2001: Die Bedeutung von Vertrauen bei der Wahrnehmung und Bewertung von Risiken. In: Akademie für Technikfolgenabschätzung in BadenWürttemberg (Hg.): Arbeitsbericht, Nr. 197. Stuttgart Slovic, P., 1987: Perception of Risk. In: Science 236 (1987), S. 280-285

Torgersen, H.; Hampel, J. et al., 2002: Promise, Problems and Proxies: Twenty-five Years of Debate and Regulation in Europe. In: Bauer, M.; Gaskell, G. (Hg.): Biotechnology. The Making of a Global Controversy. Cambridge, S. 21-94

Torgersen, H.; Hampel, J., 2012: Calling Controversy: Assessing Synthetic Biology's Conflict Potential. In: Public Understanding of Science 21/2 (2012), S. $134-148$

Weitze, M.D.; Heckl, W., 2016: Wissenschaftskommunikation. Schlüsselideen, Akteure, Fallbeispiele. Berlin Weyer, J.; Kroninger, J.; Hoffmann, S., 2012: Technikakzeptanz in Deutschland und Europa. In: Priddat, B.; West, K.-W. (Hg.): Die Modernität der Industrie. Marburg, S. 317-356 
Zapf, W.; Breuer, S.; Hampel, J., 1986: Technikfolgen für Haushaltsorganisation und Familienbeziehungen. In: Lutz, B. (Hg.): Technik und sozialer Wandel. Verhandlungen des 23. Deutschen Soziologentages in Hamburg 1986. Frankfurt a. M., S. 220-232

Zwick, M.M., 1990: Neue soziale Bewegungen als politische Subkultur. Zielsetzung, Anhängerschaft, Mobilisierung - Eine empirisches Analyse. Frankfurt a. M.

Zwick, M.M., 1998: Wertorientierungen und Technikeinstellungen im Prozeß gesellschaftlicher Modernisierung: das Beispiel der Gentechnik. Arbeitsbericht 106 der Akademie für Technikfolgenabschätzung in Baden-Württemberg. Stuttgart

Zwick, M.M.; Renn, O., 1998: Wahrnehmung und Bewertung von Technik in Baden-Württemberg. Akademie für Technikfolgenabschätzung in Baden-Württemberg. Stuttgart

Zwick, M.M.; Renn, O., 2002: Wahrnehmung und Bewertung von Risiken. Ergebnisse des Risikosurvey Baden-Württemberg 2001. Arbeitsbericht Nr. 202 der Akademie für Technikfolgenabschätzung in BadenWürttemberg. Stuttgart

Zwick, M.M.; Renn, O., 2008: Risikokonzepte jenseits von Eintrittswahrscheinlichkeit und Schadenserwartung. In: Felgentreff, C.; Glade, T. (Hg.): Naturrisiken und Sozialkatastrophen, Berlin, S. 77-98

\section{Kontakt}

Dr. Jürgen Hampel

E-Mail: juergen.hampel@sowi.uni-stuttgart.de

Dr. Michael M. Zwick

E-Mail: michael.zwick@sowie.uni-stuttgart.de

Institut für Sozialwissenschaften

Universität Stuttgart

Seidenstr. 36, 70174 Stuttgart

$《 》$

\section{Partitionierung und Transmutation: Eine kerntechnische Zukunftsoption?}

\author{
von Diana Gallego Carrera und Michael \\ Ruddat, DIALOGIK gGmbH und Zentrum \\ für interdisziplinäre Risiko- und \\ Innovationsforschung (ZIRIUS), Universität \\ Stuttgart
}

Wärmeentwickelnde, hochradioaktive Abfälle haben ein hohes Langzeit-Gefährdungspotenzial. Sie müssen in einem möglichst sicheren Endlager verwahrt werden, das es derzeit in Deutschland noch nicht gibt. Durch Partitionierung und Transmutation (P\&T) könnte die Trennung der Abfälle (Partitionierung) sowie die Umwandlung (Transmutation) eines Großteils der Radionuklide durch Neutronenbeschuss gelingen (Knebel et al. 2013; Lübbert/Ahlswede 2008). P\&T könnte somit eine Möglichkeit sein, das benötigte Volumen für ein Endlager für hochradioaktive Abfälle zu verringern. Bislang lag der wissenschaftliche Fokus bei P\&T auf technischen Machbarkeitsstudien. In einem interdisziplinären Forschungsprojekt wurden nun erstmals auch die gesellschaftlichen Implikationen dieser technologischen Anwendungen untersucht. Anhand von vier Szenarien (Abstinenz, Forschungspartizipation, Europäische Systempartizipation, Anwendung in Deutschland) werden im Folgenden Chancen und Risiken von P\&T beschrieben und potenzielle Handlungsmöglichkeiten sowie Kommunikationsempfehlungen formuliert. ${ }^{1}$

Heat-generating high-level radioactive waste is potentially dangerous for a very long time. It has to be stored in a high security repository, which doesn't exist today in Germany. The technology of Partitioning and Transmutation (P\&T), which is to convert part of the longlived high-level radioactive substances contained in the spent fuel rods into shorter-lived fission products (Knebel et al. 2013; Lübbert/Ah/swede 2008), is currently under research and development. It could be one way of reducing the long-term hazard potential of heat-producing waste. Until recently, technical aspects dominate the scientific debate about $P \& T$. Social implications of P\&T were now at the 